\title{
HUMAN SAFETY IN A MAN-MADE ECOSYSTEM
}

\author{
G.R. KAMALETDINOVA, M.P. ONEVSKY \& S.A. SKVORTSOV \\ Scientific laboratory of design and modelling of complex engineering systems and the Department of information \\ processes and control, Tambov State Technical University, Russian Federation.
}

\begin{abstract}
The human health condition and operating capability depend on a variety of environmental factors. It is of great importance to perform studies addressing these factors and their influence on humans as it can help to form an optimal strategy in emergency situations and help humans to attain maximum performance. The best results are shown in studies performed in isolation in a man-made ecosystem because in this case environmental processes are defined and controlled. Man-made ecosystems include a life support system (LSS) combined with the environment and a key element - human.

The research defines these three components as a complex system. This study is dedicated to interaction analysis and the human safety in this system, especially in case of emergencies. It includes supporting simulation models based on two types of experiments conducted by the researchers, namely the studies on the mean time to failure (MTF), and on human behavior during emergencies. The basic imitators of life support systems are complemented by additional environmental models where a human is represented as a loading element. To prevent hazards in low performance situations, the simulator used during the experiment, had a decision-making system, controlling environmental parameters and taking actions for the stabilization. It could also control the human condition, such as pulse, pressure, etc., during the entire operation. The results of the studies showed the dependency of human performance on fatigue, the time spent in isolation, as well as the emergencies. The methodology recommendations and safety requirements are discussed in the paper. The results of these studies were used in forming an information database for automated control system of the "artificial lungs" equipment.
\end{abstract}

Keywords: hazard, isolation studies, life support system, man-made ecosystem, simulation

\section{INTRODUCTION}

Human life depends on environmental factors. Disasters, ecological problems or even smaller emergencies can cause performance reduction, as well as serious health problems. There are many studies concerning ecological factors and their influences on humans. However, modern ecosystems in the cities are becoming more and more unnatural: climate regulation, air/ water reclamation, utilization of natural sources etc Thus, it is important to look into studies on man-made ecosystems because they allow the exploration of environmental factors and their influence on humans in greater details.

Researches on man-made ecosystems usually focus on submarines or aerospace systems because, due to their isolation, they model the natural environment on a higher level. However, the interest in deep space manned missions, and the technology development used for the missions, increase the number of ecological problems. This raises the importance of additional experiments and studies aimed in solving engineering problems and increasing general health and safety. To achieve these goals, experiments have to be prolongated, they have to include human factor, and also provide a certain level of isolation.

Experiments examined in this article are based on real simulation. Real-time models of LSSs are combined with additional environmental models, such as a model of a human and a model of system service. The models are based on the practice of operating aerospace LSS, environmental studies, and "reference man" model. This approach was used during ground isolation experiments and during a series of additional experiments at university. 


\section{METHODOLOGY}

\subsection{General methodology}

A man-made ecosystem is significantly different, compared to a natural ecosystem. Human is a decision-making element and his/her safety is a key issue but at the same time it is important to control human's actions in case of an emergency. The impact of stress can affect the human control. Moreover, there should be a duplication of elements (for example, elements of the oxygen generation system or duplication of the power supply system) for higher safety. The same approach is used, for example, in aviation.

A man-made ecosystem is created and organised from man-made elements and the number of elements should be limited [1]. All of them should be connected by energy exchanges and transformation of mass flow. Moreover, it should be stressed out, that the processes in a manmade ecosystem are more intensive than the transformation of natural processes. Signals exchange in the man-made ecosystem and its interaction with human is based on Anokhin's theory [2], explaining how signals and the following reactions are related. The most important elements of the system are shown in Fig. 1. The system includes:

- The "COMPLEX" - this element includes simulation models describing LSSs, environment and human, signals and data synthesizing and processing and also a model of "decision support system" which analyses functioning conditions and can automatically perform actions aimed to fix malfunctions of a sub-system if possible.

- The "HUMAN" - this element provides a person working with the system (an operator) with tools (software or hardware) helping to perform maintenance activities, malfunction analysis, manual activities, etc. It includes parameters of the experiments participants.

- The "CONTROL" - this element evaluates human performance. During experiments, it is also responsible for malfunction settings (type of emergencies, levels of danger and difficulty) and manual operations in case of low human performance.

\subsection{Approach to the modelling}

Imitation modelling was chosen as an approach for system development as it seems to be the best solution for the complex systems design. The modelling requires a real-time approach so it could be possible to perform maintenance activities, supervise systems, fix malfunctions, and understand their influence on safety and system sustainability [3].

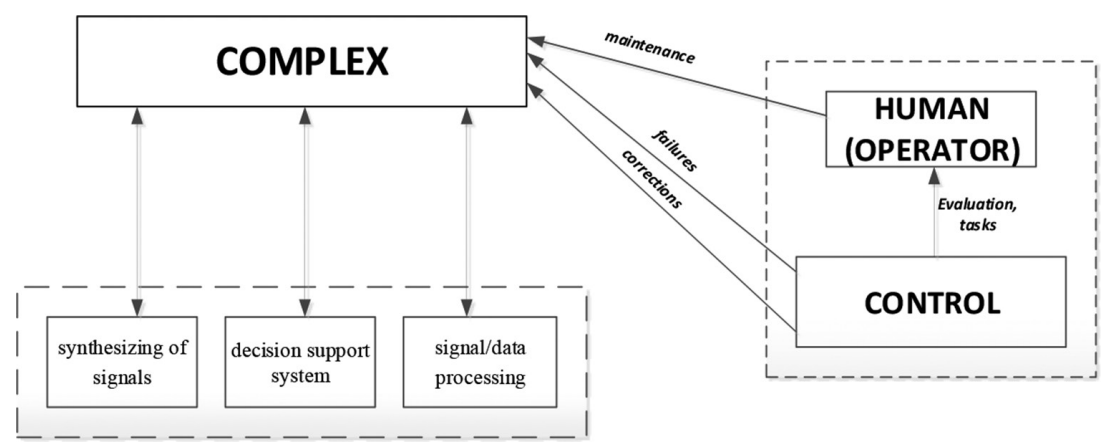

Figure 1: Organisation of the interaction. 


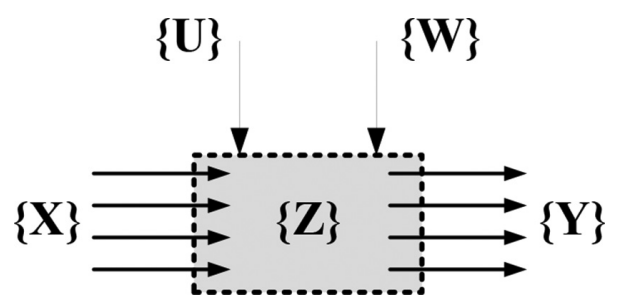

Figure 2: State description of models.

LSSs models were described with following states (Fig. 2): input $\{X\}$, output $\{Y\}$, internal $\{Z\}$ and control parameters $\{U\}$ and internal and external disturbances $\{W\}$, in which the input, output, and control parameters correspond with the modelling systems. External disturbances change with time and depend on environmental parameters, humans (as a result of metabolism and its products' transformation), and other systems. Internal disturbances, on the other hand, are controlled by the control parameters of modelling system. The transformation of input parameters to output parameters forms internal parameters

To form a formal mathematical description of every system, basic assumption was taken into account. Most of them are standard for the atmosphere, wherein "air" is defined as a mix of ideal gases and clear chemical compounds. Total mixture pressure does not depend on trace pollutants, which are consisted of basic elements [4-5] such as spirits, aldehydes, ketones, hydrocarbons, acetones, nitrogen compounds, acids, and inorganic compounds. Mass flows of atmosphere and trace pollutants are calculated on every step of integration. To create a human model, an international "reference man" was used [1], wherein mass flow and heat-transfer intensity are proportional to energy expenditure.

Proportionality factor was corrected based on simulation experiments on a detailed model of mass and energy exchange where with the real data the exchange processes were modelled.

LabVIEW software was used in designing as it has the necessary modelling tools, providing users with visualisation, and allowing integration with hardware equipment, sensors, and monitors. The structure of the developed system includes: power sub-system linked with all other systems as every system requires a certain amount of energy; thermoregulation sub-system which has a decision support option, models of LSSs, model of environment, human model, a logical model of failure settings which is related to the CONTROL element (see in Fig.1) [6-9]. Verification of the models was based on algorithm tests on contradictoriness, results correlation analysis and ergonomic evaluations.

\section{EXPERIMENTS}

Two types of experiments were conducted, namely studies on the mean time to failure and on human behaviour during emergency situations.

\subsection{Mean time to failure}

As the LSSs models are based on the actual solutions, it was known in advance that some parts of the equipment had limited resources. For example, filters, regenerating cartridges, water supply (tanks) or different gas balloons. Resources are limited by the volume of the tanks/balloons or by the time of usage (as filters or cartridges collect waste). LabView software allows connection to the hardware devices and it is possible to run in parallel two models: full simulator and the same model with a connected hardware part. 
Based on this methodology, the experiment had two goals: (1) to compare data received from simulation experiment and from hardware; (2) to analyze how long the hardware will work until a failure will appear and to compare these results with the data gathered from existing literature [9-10].

\subsubsection{Results}

Comparison of research outputs in existing literature with certificates of filters/cartridges, and with hardware simulation, showed that the producer usually lowers resources (up to $15 \%$ ) which gives additional safety to the equipment in the worst case scenario (for example in case of polluted water and water filters). In the situation with gas balloons, the simulation showed a longer resource comparing with the hardware as there can be a leakage and to spend all the gas in the balloon is not possible.

Based on these results, simulation models were corrected, so human behaviour could be analysed in precised simulators. Moreover, these experiments helped to check the resources and characteristics of new equipment (as it was done in studies dedicated to regenerating cartridges analysis), as well as to collect experimental data and to ensure human safety.

\subsection{Human behaviour in emergencies}

Analyses of human behaviour and human performance during emergency situations are based on isolation studies with human participation $[1,11]$ and on additional experiment on base of university.

Based on the MTF analysis, situations of resources expiration were simulated. Moreover, literature review (including [9] and [10]) indicated some typical hardware failures - the situation when the hardware was physically broken (for example a heating element could be damaged). Such cases were also simulated. At the same time, the man-made ecosystem needed maintenance activities from operators as day and night cycles were different.

Consequently, the system allowed simulating maintenance activities, resource limitations, failures related to equipment damages, and extreme emergencies such as fires, depressurization, or power system malfunction.

These experiments included human interaction with the system. Internal experiments were focused on ergonomics and the MTF analysis, preliminary studies and on data collecting. The main part of experiment had 3 stages of isolation: 14 days, 105 days and 520 days. Every time six people, carefully selected by medics and psychologists, were isolated. Every member of the group had his own schedule of system monitoring and maintenance. "Control" element (figure 1) set up failures equally between the members and following the protocol.

If system parameters changed due to a malfuction or wrong operation and there was no human intervention, the system would support automatic regulation, which is called decision-making system. However, this situation would be seen on the protocol as an automatic regulation and would influence the human performance analysis.

To examine human behaviour and performance, different methods were used:

- Every member of the group had different surveys where they were asked about the usability of the software and monitors.

- It was possible to supervise their activities, to analyze operator's carefulness, accuracy and overall behavior.

- Thanks to the special biomedical device installed in a computer mouse, heart rhythm parameters, pressure and rhythm variation (rhythmogram) were measured. 
- All the malfunctions were ranked by its complexity and scheduled in advance. All the protocols and schedules were available for analysis afterwards.

- System had a timer analyzing how long the operator was working with a system (in case of a failure elimination or changing of hardware, or in case of item expiration).

The following analyses were conducted:

- Based on ergonomic factors, sufficiency analysis of controlling parameters was conducted to determine whether the level of demonstrativeness is satisfactory in order to consider the level of safety to be appropriate.

- Visual non-reliance analysis.

- Analysis, through comparison, of human psychophysiological state in emergencies and maintenance activities performed with a special biomedical device.

- Analysis of effectiveness of actions taken to prevent and fix failures, which are based on the approach used in trainings for pilots and astronauts [11-14].

- Statistic safety and execution analysis based on complexity of failures and the outcome [11].

To provide a certain level of safety and sustainability, experimental statistic performance evaluations $\hat{x}_{k}$ should meet the following requirements:

$$
\begin{gathered}
\mathrm{P}_{\text {safety }}=\mathrm{P}_{\mathrm{x}}\left(\mathrm{U}<\mathrm{U}_{\text {add }}, \pi(\Delta \hat{\mathrm{Y}})<\pi_{1}(\Delta \hat{\mathrm{Y}})\right) \geq 0,995, \\
\mathrm{P}_{\text {exec }}=\mathrm{P}_{\mathrm{x}}\left(\mathrm{U}<\mathrm{U}_{\text {add }}, \pi(\Delta \hat{\mathrm{Y}})<\pi_{2}(\Delta \hat{\mathrm{Y}})\right) \geq 0,95,
\end{gathered}
$$

where $\mathrm{P}_{\text {safety }}\left(\mathrm{U}<\mathrm{U}_{\text {add }}, \pi(\Delta \hat{\mathrm{Y}})<\pi_{1}(\Delta \hat{\mathrm{Y}})\right)$ is the probability value of human safety in emergencies, $\mathrm{P}_{\text {exec }}\left(\mathrm{U}<\mathrm{U}_{\text {add }}, \pi(\Delta \hat{\mathrm{Y}})<\pi_{2}(\Delta \hat{\mathrm{Y}})\right)$ is the probability value of execution in all operating basis failures, $\pi_{1}(\Delta \hat{\mathrm{Y}}), \pi_{2}(\Delta \hat{\mathrm{Y}})$ is the loss causing critical situations for humans, and $\mathrm{Y}$ is the outcome of failures with complexity $\mathrm{U}$.

\subsubsection{Results}

The said experiments were conducted through ground isolation studies [1]. Preliminary experiments on base of university and 14-days isolation experiment were mainly focused on the ergonomic analysis and visual analysis. These preliminary studies helped to determine and adjust methodology to the main part of studies.

During 105 days and 520 days of isolation, the human (the operator) fixed more than 100 scheduled failures and conducted daily (morning and evening) maintenance activities. The workload was equal for every human involved in the experiment. The variation of human performance was analysed. These results are shown in Fig. 3.

The figure shows human performance within normal limits with points of instability that are attributable to the fatigue factor. Fatigue and stress in emergencies play crucial role in safety. As shown in Fig. 4, the human performance decreased, and it can be observed that in this situation, the operator needs more time to fix a failure. 


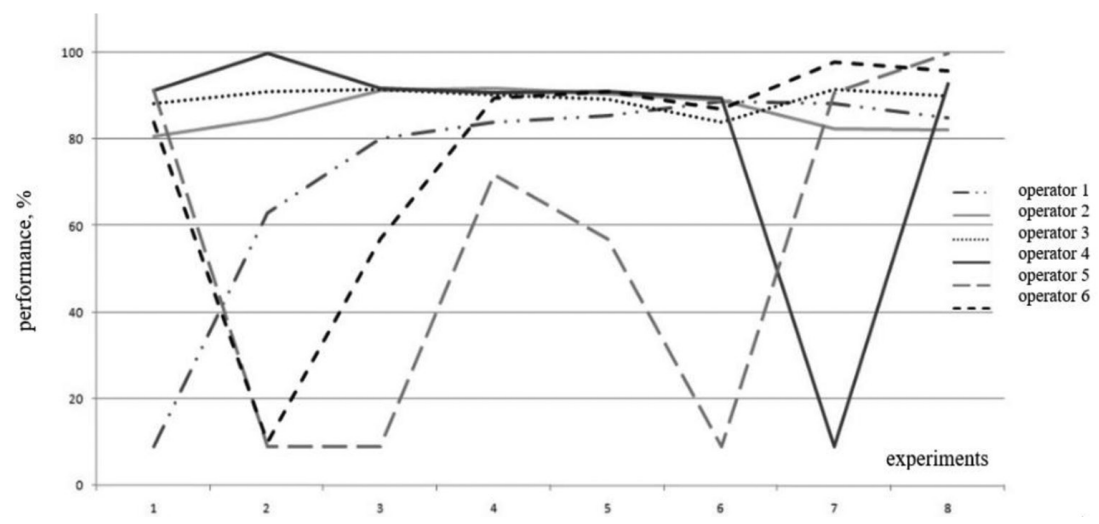

Figure 3: Human performance.

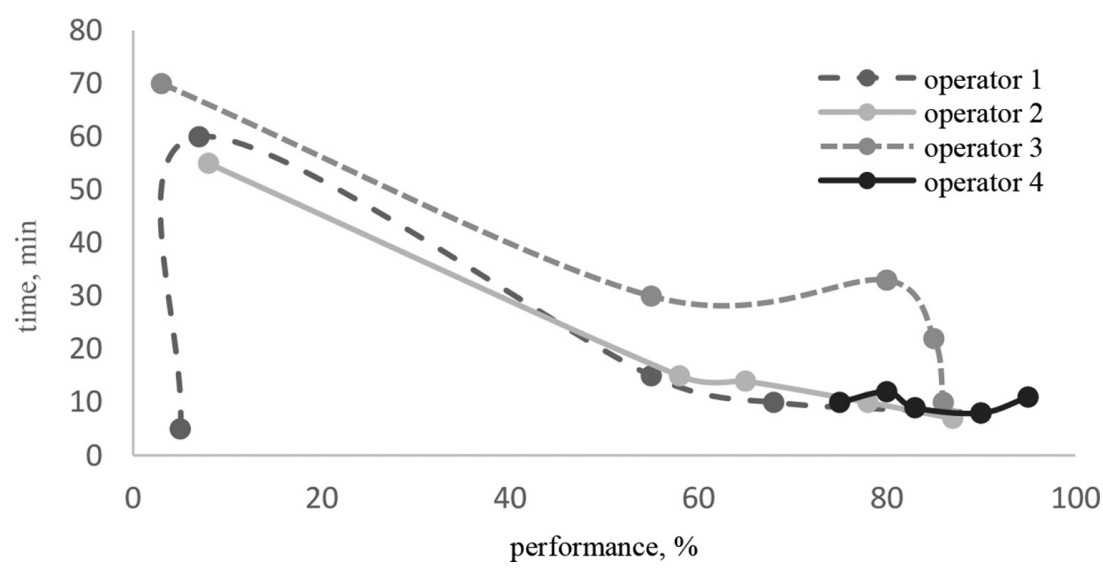

Figure 4: Dependency of fixing the failure from the level of performance (4 operators).

Based on the presented approach (1), (2), the probability value of human safety was calculated as $\mathrm{P}_{\text {safety }}>0,995$. The probability value of execution in all operating basis failures following the experiments are $P_{\text {exec }}>0,9259$ - for 105 days of experiment and $P_{\text {exec }}>0,952$ for 520 days of experiment.

\section{CONCLUSIONS}

The performed experiments helps to understand the interaction between human and man-made ecosystems, to analyse human safety, and to understand the connection between human performance and actions during emergencies. The following conclusions were drawn from the study:

- An expanded simulation model of a man-made ecosystem was designed;

- The methodologies of the experiments and analysis were developed;

- Reports given by experiment participants were utilized to improve the ergonomic properties of the system;

- Thanks to the hardware testing, aknowledge information database was created;

- Isolation experiments helped in examination of the human behaviour in daily routine and emergency situations and to evaluate human performance and safety. 


\section{ACKNOWLEDGEMENTS}

This work was supported by the Russian Science Foundation (Agreement number 15-19-10028).

\section{REFERENCES}

[1] Kurmazenko, E.A., Khabarovskiy, N.N., Kamaletdinova, G.R., Demin, E.P. \& Morukov, B.V., Life Support System Virtual Simulators for Mars-500 Ground-Based Experiment, ed. D.N. Ghista, Biomedical Science, Engineering and Technology, InTech: Croatia, pp. 535-558, 2011.

[2] Anokhin, P.K., Fundamental questions functional systems theory. Principles of functional systems organization, Nauka: Moscow, pp. 5-61, 1973. (in Russian)

[3] Hemdi, A., Introduction to operations research theory. Part 18. Imitation modeling, Williams: Moscow, pp. 697-737, 2007. (in Russian)

[4] Kurmazenko, E.A., Functional Analysis of Life Support Systems. Tutorial, NIIchimmash: Moscow, 1997. (in Russian)

[5] Umerlagin, T.G., Mathematical Modeling of Basic Chemical-Engineering Processes. Part 1, UGNTU: Ufa, 2001. (in Russian)

[6] Belz, S., Detrell, G., Ganzer, B. \& Messerschmid, E., Synergetic hybrid life support system for a mars transfer vehicle. Proceedings of 61 st International Astonautical Congress, pp. 7896-7906, 2010.

[7] Czupalla, M., Zhukov, A., Schnaitmann, J., Bickel, T. \& Walter, U., The virtual habitat: a tool for dynamic life support system simulations. AIAA Technical Paper Series, pp. 6016-6028, 2010.

[8] Nakanel, M., Yamazaki, N., Ishikawa, Y. \& Miyajima, H., Autonomous control method for material circulation in advanced life support system. AIAA Technical Paper Series, pp. 6227-6232, 2010.

[9] Jones, H., Life support with failures and variable supply. AIAA Technical Paper Series, pp. 6238-6257, 2010.

[10] Bobe, L.S., Samsonov, N.M., Gavrilov, L.I., et al., Regenerative life support systems experience on board of "Salut", "MIR", "ISS" space stations. Aerospace and Ecological Medicine, 6(1), pp. 10-12, 2008. (in Russian)

[11] Sokhin, I.G., Adaptive-competence approach to the problem of quality control of cosmonaut training in the frames of space safety and sustainability guarantee. Human Spaceflight, 1(3), pp. 36-48, 2012. (in Russian)

[12] Zinchenko, V.P., Mayzel, N.I., Nazarov, A.I. \& Tsvetkov, A.A., Analysis of humanoperator activity, Industrial psychology: Moscow, 1964. (in Russian)

[13] Talalaev, A.A., Psychophysiological monitoring of the population functional state as a part of Russian national security system, NeuroLab: Moscow, 2010. (in Russian)

[14] Baevsky, R.M. \& Berseneva, A.P., Assessment of adaptive capabilities and the risk of diseases development, Medicine: Moscow, 1997. (in Russian) 